\title{
Assessment of Toxicity of Cdse/Cds/Zns/S,S-Dihydrolipoic Acid/Polyacrylic Acid Quantum Dots at Danio rerio Embryos and Larvae
}

\author{
Konstantin V. Zolotarev, ${ }^{1}$ Valentina N. Kashirtseva, ${ }^{1}$ Alexey V. Mishin, ${ }^{2}$ Natalya F. Belyaeva, ${ }^{1}$ \\ Natalya V. Medvedeva, ${ }^{1}$ and Olga M. Ipatova ${ }^{1}$ \\ ${ }^{1}$ Institute of Biomedical Chemistry, Russian Academy of Medical Sciences (IBMC RAMS), 10 Pogodinskaya Street, \\ Moscow 119121, Russia \\ ${ }^{2}$ EcoBioPharm LLC., 11-1 Bolshoy Kozlovsky Lane, Moscow 107078, Russia
}

Correspondence should be addressed to Natalya F. Belyaeva, natalia.belyaeva@ibmc.msk.ru

Received 10 April 2012; Accepted 29 May 2012

Academic Editors: Y. Jin and B. R. Kimball

Copyright ( $) 2012$ Konstantin V. Zolotarev et al. This is an open access article distributed under the Creative Commons Attribution License, which permits unrestricted use, distribution, and reproduction in any medium, provided the original work is properly cited.

\begin{abstract}
Quantum dots (QDs) are nanosized semiconductor crystals. They are currently applied in different science fields such as medicine, namely, cancer diagnostics and treatment. QD toxicity is caused by the toxicity of their components. In vivo application of QDs requires their toxicity assessment, so the purpose of this work has been the estimation of acute and chronic toxicity of the QDs at Danio rerio embryos and larvae, QDs being composed of CdSe/CdS/ZnS/S,S-dihydrolipoic acid/polyacrylic acid. We have found no QD acute toxicity during 48 hours of QDs action at the embryo up to the concentration of $185 \mu \mathrm{M}$ Cd. QDs have been found to be toxic only at 5-7 days of action, it shows that QDs act accumulatively. Beside lethality, we have observed different larval development defects, that is, differently localized edemas, lag of development, tail curvature, and swimming bladder malformation. Our experimental data as well as literature data show that toxicity of the quantum dots at Danio rerio embryos and larvae is primarily caused by toxic action of $\mathrm{Cd}^{2+}$ ion which arises from partial dissociation of CdSe and CdS molecules.
\end{abstract}

\section{Introduction}

Quantum dots are more and more applied in different fields of science and technology nowadays due to their specific semiconductive optical properties. High luminescence of QDs makes them applicable for high-resolution analytic or diagnostic purposes. The most widespread QDs are those which include cadmium selenide (CdSe) or cadmium telluride (CdTe). The QDs are notable because it is possible to adjust some biological markers to their surface. Nowadays, much attention is paid to QDs application in oncology, namely, cancer diagnostics and treatment [1]. The QDs application for finding and visualization of a tumor is based on their ability to be accumulated in a spread vascular system of a tumor when injected into a body [2]. Nanovalues of sizes, narrow fluorescence peaks high-fluorescence intensity being several tens greater than that of organic fluorophores traditionally used for visualization of cell inner compartments, are the useful specifications of the quantum dots.

So the QDs with a somehow modified surface may be used for living tissue researches with some kinds of microscopy. The QDs are widely applied in biomedical researches nowadays, for example, in visualization of some cell surface receptors, cytoskeleton components, antigens, and so forth. Wu et al. [3] have shown an opportunity to observe 2 targets on a cell surface at the same time using QDs with different spectra, dots surface being modified with immunoglobulin and streptovidin.

Lim et al. [4] have used theoretical and experimental researches to show that one should take a target tissue properties into consideration when using QDs. The dots are chemically active that may negatively affect the results of researches using them. It should be mentioned that QD toxicity and biocompatibility as well as the fate of injected 


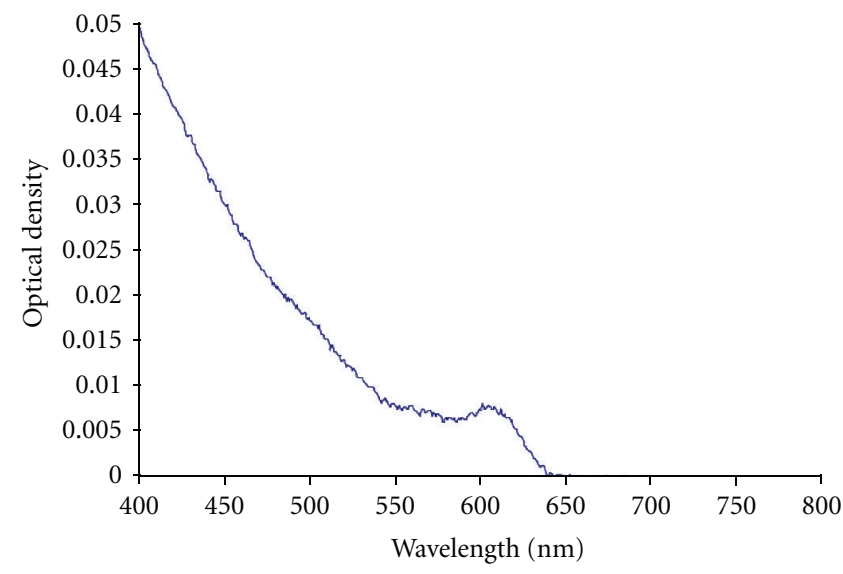

(a)

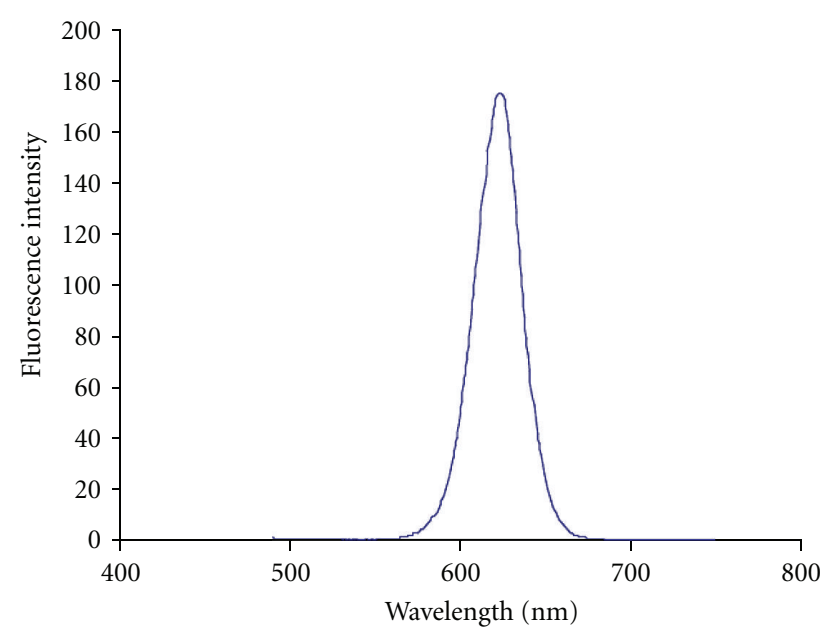

(b)

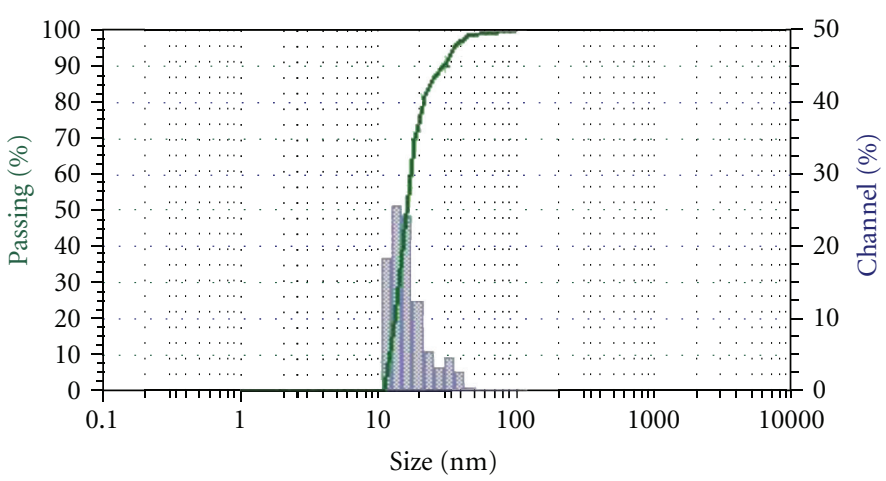

(c)

FIGURE 1: The tested quantum dots specifications: (a) absorbance spectrum, (b) fluorescence spectrum, (c) particle size distribution.

into the body QDs are notably important. In order to solve these problems, the QDs are provided with a special coating that prevents living tissues from harmful action and saves the QD properties necessary for the research.

In vivo application of QDs requires their toxicity assessment, so the purpose of this work has been the assessment of acute and chronic toxicity of the quantum dots using Danio rerio embryos and larvae, QDs being composed of CdSe/CdS/ZnS/S,S-dihydrolipoic acid/polyacrylic acid. Beside that, those QDs cardiotoxicity and teratogenicity assessment have been made. Danio rerio (i.e., zebrafish) has been applied as a laboratory model for assessment of different chemicals toxicity since 1950s. This model may be considered to be an economy and handy test object for an intermediate research between after testing at a cell culture and before testing at a mammal model [5-7].

\section{Methods of the Research}

The CdSe/CdS/ZnS/S,S-dihydrolipoic acid/polyacrylic acid QDs used for the toxicity assessment have been produced by the Research Institute for Applied Acoustics (Dubna, Moscow region, Russia). Some properties of those QDs are shown on Figure 1.
According to the producer's data, the average particle size of a QD is $18.5 \mathrm{~nm}$ and the cadmium amount in QDs is about 3700 moles of Cd per 1 mole of QDs.

The test objects for the toxicity assessment have been the embryos and larvae of a wild type of Danio rerio. We used sexually mature fishes with age from 6 to 12 months to get the embryos. Fishes were put into reconstituted water according to an ISO standard [8] in an aquarium with appropriate conditions $\left(26 \pm 1{ }^{\circ} \mathrm{C}\right.$ temperature, 14-hour daylight, water filtration, and airing). Males and females were put with a $1: 2$ ratio into water which volume in liters was equal to total number of fishes. A net with mesh of maximum $2 \mathrm{~mm}$ size was put onto the aquarium bottom not to let the fishes eat their eggs. Spawning usually took place in the beginning of the next daylight time. At 3-4 hours after spawning fishes were removed from the spawning aquarium, the net and excess water were removed too. Eggs were transported into a Petri dish with reconstituted water using pipettes with cut tips. Then fertilized undamaged eggs were selected using a Leica EZ4D binocular microscope and transferred into another Petri dish [9].

2.1. Assessment Procedure. The tested QD suspensions were prepared in the wells of 24 -well plastic microtiter plates, 


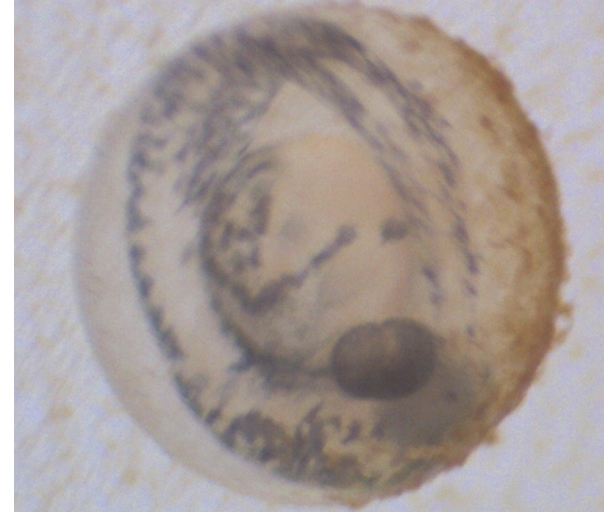

(a)

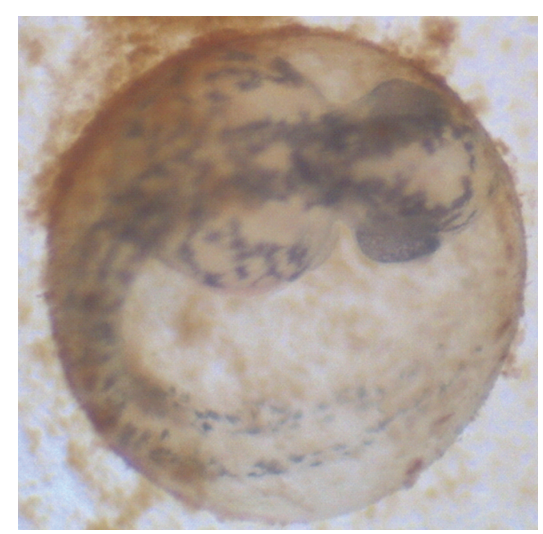

(b)

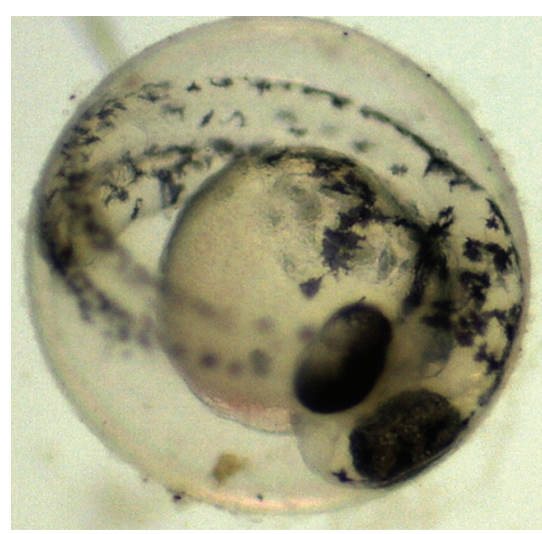

(c)

FIGURE 2: Zebrafish embryos having been developing normally for 48 hours in: (a) the QD suspension with a Cd concentration of $35 \mu \mathrm{M}$, (b) the QD suspension with a Cd concentration of $185 \mu \mathrm{M}$, (c) the control. Zoom factor is 35.

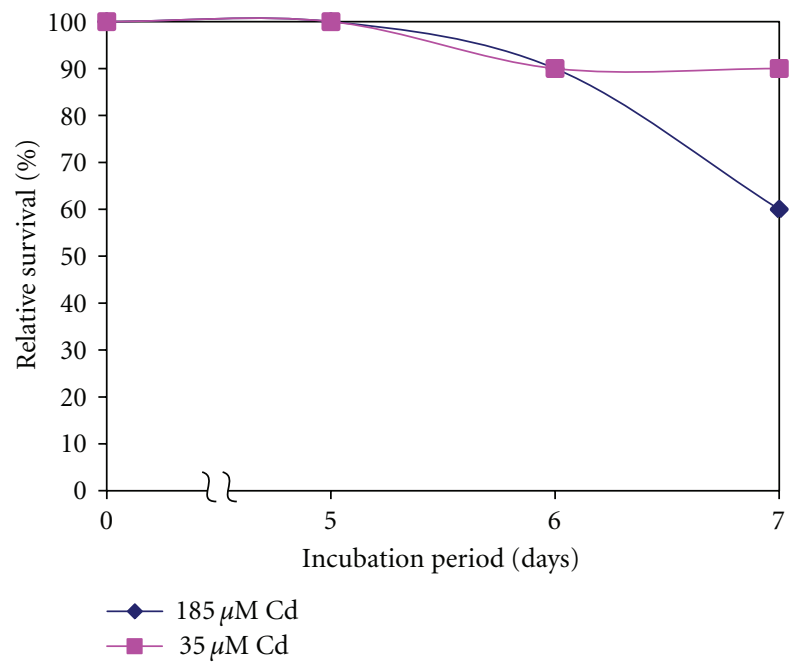

FIGURE 3: Graph of average survival of the larvae versus the QD concentration, survival being divided by that of control.

suspension volumes being $2 \mathrm{~mL}$ per well. The primary substance prepared by the producer was diluted by the reconstituted water preliminarily aired. After that 1 , selected egg was placed into each well. Wells of a control plate were loaded with $2 \mathrm{~mL}$ of the aired reconstituted water and 1 egg per each too. All the plates were then put into a temperature stating Binder BD23 incubator with a passive ventilation at a $26 \pm 0.1^{\circ} \mathrm{C}$ temperature.

2.2. Quantum Dot Acute Toxicity Assessment. After 24 and 48 hours of incubation, dead embryos amount per each QD concentration or control was calculated and registered. An embryo is considered to be dead if it is either opaque, or its development has stopped, or it has no heartbeat at 48 hours, opaqueness being the most typical indication. In order to assess the acute toxicity, we made a graph of average survival rate of the embryos versus common logarithm of the QD concentration, survival being divided by that of control.
Using this graph, we figured out the value of QD concentration at $50 \%$ survival if possible (i.e., $\mathrm{LC}_{50}$ ).

\subsection{Quantum Dot Cardiotoxicity and Teratogenicity Estima-} tion. After 48 hours of incubation, we assessed QD teratogenicity. In order to do that, we calculated the amounts of the defects of embryonic development (i.e., head, tail, chord malformations), yolk sac injures, lag of development, and so forth. The defects were illustrated using a digital camera built in the Leica EZ4D binocular microscope. At the same time, QD cardiotoxicity was also assessed, pericardial edemas and heartbeat rate being calculated for this purpose. Heartbeat rate values at each QD concentration were averaged and compared with control ones.

2.4. Quantum Dot Chronic Toxicity Estimation. After 6 and 7 days of incubation, QD chronic toxicity was assessed. In order to do that, we calculated the amounts of the defects in larval development (i.e., body shape change, tail curvature, lag of development, edemas, behavior violations-too frequent breath, bad swimming coordination, low motive activity), and also dead larvae amount. A larva is considered to be dead if it either does not move, or does not react on mechanical disturbance, or has no heartbeat. The defects of larval development were also illustrated using the built-in digital camera.

\section{Results and Discussion}

3.1. Quantum Dot Toxicity Assessment. We have found no QD toxicity up to the concentration of $185 \mu \mathrm{M} \mathrm{Cd}$. We have also observed neither cardiotoxic nor teratogenic effects in the tested Cd concentration interval of the QDs (i.e., 35$185 \mu \mathrm{M} \mathrm{Cd}$ ). Figure 2 shows normally developing embryos in QD suspensions and in controls.

The results of quantum dot chronic toxicity assessment are shown in the Table 1, larval survival being shown on Figure 3. According to Figure 3, Danio rerio larvae survival depends on Cd concentration in QDs and on duration of the QD action. 


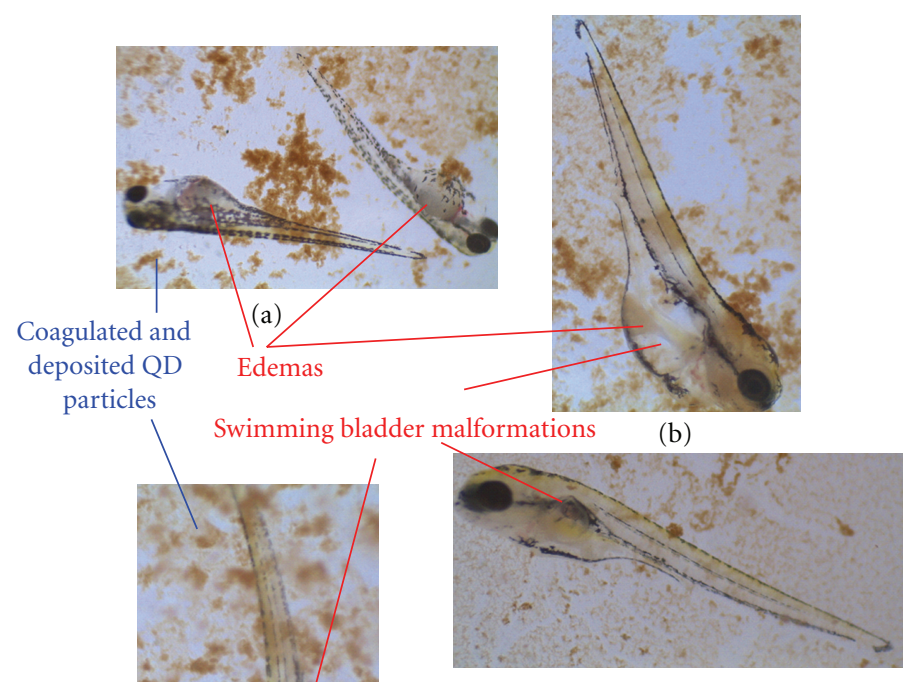

(d)

FIGURE 4: Zebrafish larvae with developmental defects after 7 days of incubation in QD suspensions with Cd concentration of $185 \mu \mathrm{M}$ (a-c) and of $35 \mu \mathrm{M}$ (D). (a) with an edema in QD suspension, (b) with a large edema and a swimming bladder malformation, (c) with a lag of development, a curvature and a swimming bladder malformation, (d) with a swimming bladder malformation. Zoom factor is 30 .

TABLE 1: Quantum dot chronic toxicity assessment at zebrafish larvae.

\begin{tabular}{|c|c|c|c|c|}
\hline \multirow{3}{*}{$\begin{array}{l}\text { Concentration of } \\
\text { Cd in QDs, } \mu \mathrm{M}\end{array}$} & \multicolumn{4}{|c|}{ Average amount of larvae, $\%$, having } \\
\hline & \multicolumn{2}{|c|}{ Sublethal effects } & \multicolumn{2}{|c|}{ Lethal effects } \\
\hline & 6 days of incubation & 7 days of incubation & 6 days of incubation & 7 days of incubation \\
\hline 0 (control) & 0 & 0 & 0 & 0 \\
\hline 35 & 0 & Edemas-30 & 10 & 10 \\
\hline 185 & Edemas-30 & $\begin{array}{l}\text { Lag of development }-10 \\
\text { Tail curvature-10 } \\
\text { Edemas- } 60\end{array}$ & 10 & 40 \\
\hline
\end{tabular}

The developmental defects at the larvae are shown on Figures 4, and 5 being for comparison.

During the assessment procedure, it was notable that QD particles coagulated (got stuck to each other) and deposited after the primary QD suspension got diluted by the reconstituted water, the latter being a solution of some inorganic salts. This process is considered to be caused by the influence of the ions of those salts, being called electrolytic coagulation of the particles. When the electrolytes are added into water, they dissolute into ions which make water molecules migrate to them, this forming solvates. It pushes the particles closer to each other and makes the chance of coagulating collision higher. The bigger particles deposit faster, depositing velocity being proportional to the square of a particle diameter according to Stokes' law. Already after 1 or 2 days of incubation, some big deposited particles were notable (Figures $4(\mathrm{a})$ and $4(\mathrm{~b}))$ in the tested QD suspensions with maximal concentration. Coagulation of the particles dropped their chance to get into chorion pores which are 500-700 nm wide according to the field emission scanning electron microscopy data [10].

Quantum dot toxicity is primarily caused by partial dissociation of weakly water-soluble CdSe and CdS salts [11] followed by formation of $\mathrm{Cd}^{2+}$ ion capable to form coordination complexes actively. $\mathrm{Cd}^{2+}$ ion is known to be able to affect the formation of reactive oxygen species in living cells [12]. Beside that it can substitute the physiologically important $\mathrm{Ca}^{2+}$ ion [13].

During our assessment procedure, we observed QD toxicity only after 5-7 days of incubation, it showing that QDs act accumulatively. This period of toxic action showup and the whole way of QD toxic action were similar to those of cadmium salt solution toxic action (see Figure 6), this also may affirm the primary contribution of $\mathrm{Cd}^{2+}$. Though 


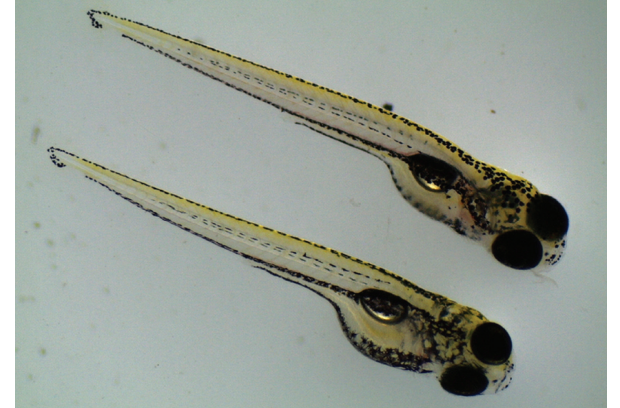

Figure 5: Healthy 7-day-old zebrafish larvae (control). Zoom factor is 30 .

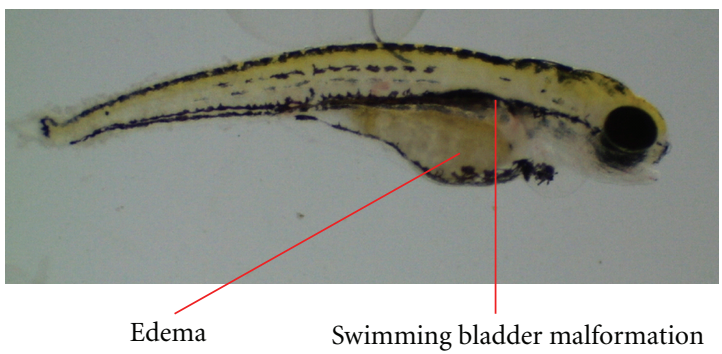

Figure 6: Zebrafish larva with an edema and a swimming bladder malformation after 5 days of incubation in a $45 \mu \mathrm{M} \mathrm{Cd}^{2+}$ solution.

it would be helpful to measure $\mathrm{Cd}^{2+}$ concentration inside $D$. rerio larvae which may give a strong evidence of the impact of the ion. We would also like to mention that King-Heiden et al. [11] have made toxicity assessment of similar Cd-based QDs at D. rerio, having expressed QD concentration as moles of Cd per liter too. According to the data of that assessment, the $\mathrm{LC}_{50}$ value after 5 days of incubation in cadmiumcontaining QD suspension varies from 7 to $42 \mu \mathrm{M} \mathrm{Cd}$, QDs having different one-layer covers. Our test has shown that the larval survival at $185 \mu \mathrm{M}$ Cd QD suspension after 7 days of incubation is a bit more then 50\% (Figure 3). The lower toxicity of our QDs expressed in Cd concentration might be supposed to be caused by the presence of a two-layer cover, the latter preventing the CdSe and CdS layers from dissociation because the cover has to dissociate first.

George et al. [14] have comparatively assessed the toxicity of CdSe/ZnS/mercaptoundecylic acid QDs and CdSe/ mercaptoundecylic acid nanoparticles. They have observed significant heartbeat rate decrease, hatching slowdown, and also death of $30 \%$ of the larvae after 5 days of their incubation in a QD suspension with $25 \mathrm{mg} / \mathrm{L}$ concentration. By the way, the toxicity of the particles without a ZnS layer was significantly higher than that of the QDs having it. This is explained by CdSe dissociation slowdown caused by the $\mathrm{ZnS}$ layer.

Thus, the results of our assessment indicate the CdSe/ $\mathrm{CdS} / \mathrm{ZnS} / \mathrm{S}$,S-dihydrolipoic acid/polyacrylic acid quantum dots toxicity at Danio rerio embryos and larvae, the toxicity being most probably caused by toxic action of a $\mathrm{Cd}^{2+}$ ion formed by partial CdSe and CdS molecules dissociation.

\section{References}

[1] X. Gao, Y. Cui, R. M. Levenson, L. W. K. Chung, and S. Nie, "In vivo cancer targeting and imaging with semiconductor quantum dots," Nature Biotechnology, vol. 22, no. 8, pp. 969976, 2004.

[2] S. Sengupta and R. Sasisekharan, "Exploiting nanotechnology to target cancer," British Journal of Cancer, vol. 96, no. 9, pp. 1315-1319, 2007.

[3] X. Wu, H. Liu, J. Liu et al., "Immunofluorescent labeling of cancer marker Her2 and other cellular targets with semiconductor quantum dots," Nature Biotechnology, vol. 21, no. 1, pp. 41-46, 2003.

[4] Y. T. Lim, S. Kim, A. Nakayama, N. E. Stott, M. G. Bawendi, and J. V. Frangioni, "Selection of quantum dot wavelengths for biomedical assays and imaging," Molecular Imaging, vol. 2, no. 1, pp. 50-64, 2003.

[5] H. Sukardi, H. T. Chng, E. C. Y. Chan, Z. Gong, and S. H. Lam, "Zebrafish for drug toxicity screening: bridging the in vitro cell-based models and in vivo mammalian models," Expert Opinion on Drug Metabolism and Toxicology, vol. 7, no. 5, pp. 579-589, 2011.

[6] N. F. Belyaeva, V. N. Kashirtseva, N. V. Medvedeva, Y. Y. Khudoklinova, O. M. Ipatova, and A. I. Archakov, "Zebrafish as a model system for biomedical studies," Biochemistry B, vol. 3, no. 4, pp. 343-350, 2009.

[7] U. Strähle and C. Grabher, "The zebrafish embryo as a model for assessing off-target drug effects," DMM Disease Models and Mechanisms, vol. 3, no. 11-12, pp. 689-692, 2010.

[8] ISO, International Organization for Standardization, "Water quality-Determination of the acute lethal toxicity of substances to a freshwater fish [Brachydanio rerio HamiltonBuchanan (Teleostei, Cyprinidae)]," ISO, 7346-3: Flowthrough method, 1986.

[9] OECD, Guideline for the testing of chemicals. Draft proposal for a new guideline. Fish Embryo Toxicity (FET) Test, 2006.

[10] D. M. Rawson, T. Zhang, D. Kalicharan, and W. L. Jongebloed, "Field emission scanning electron microscopy and transmission electron microscopy studies of the chorion, plasma membrane and syncytial layers of the gastrula-stage embryo of the zebrafish Brachydanio rerio: a consideration of the structural and functional relationships with respect to cryoprotectant penetration," Aquaculture Research, vol. 31, no. 3, pp. 325-336, 2000.

[11] T. C. King-Heiden, P. N. Wiecinski, A. N. Mangham et al., "Quantum dot nanotoxicity assessment using the zebrafish embryo," Environmental Science and Technology, vol. 43, no. 5, pp. 1605-1611, 2009.

[12] J. A. Almeida, Y. S. Diniz, S. F. G. Marques et al., "The use of the oxidative stress responses as biomarkers in Nile tilapia (Oreochromis niloticus) exposed to in vivo cadmium contamination," Environment International, vol. 27, no. 8, pp. 673679, 2002.

[13] T. Meinelt, R. C. Playle, M. Pietrock, B. K. Burnison, A. Wienke, and C. E. W. Steinberg, "Interaction of cadmium toxicity in embryos and larvae of zebrafish (Danio rerio) with calcium and humic substances," Aquatic Toxicology, vol. 54, no. 3-4, pp. 205-215, 2001.

[14] S. George, T. Xia, R. Rallo et al., "Use of a high-throughput screening approach coupled with in vivo zebrafish embryo screening to develop hazard ranking for engineered nanomaterials," ACS Nano, vol. 5, no. 3, pp. 1805-1817, 2011. 

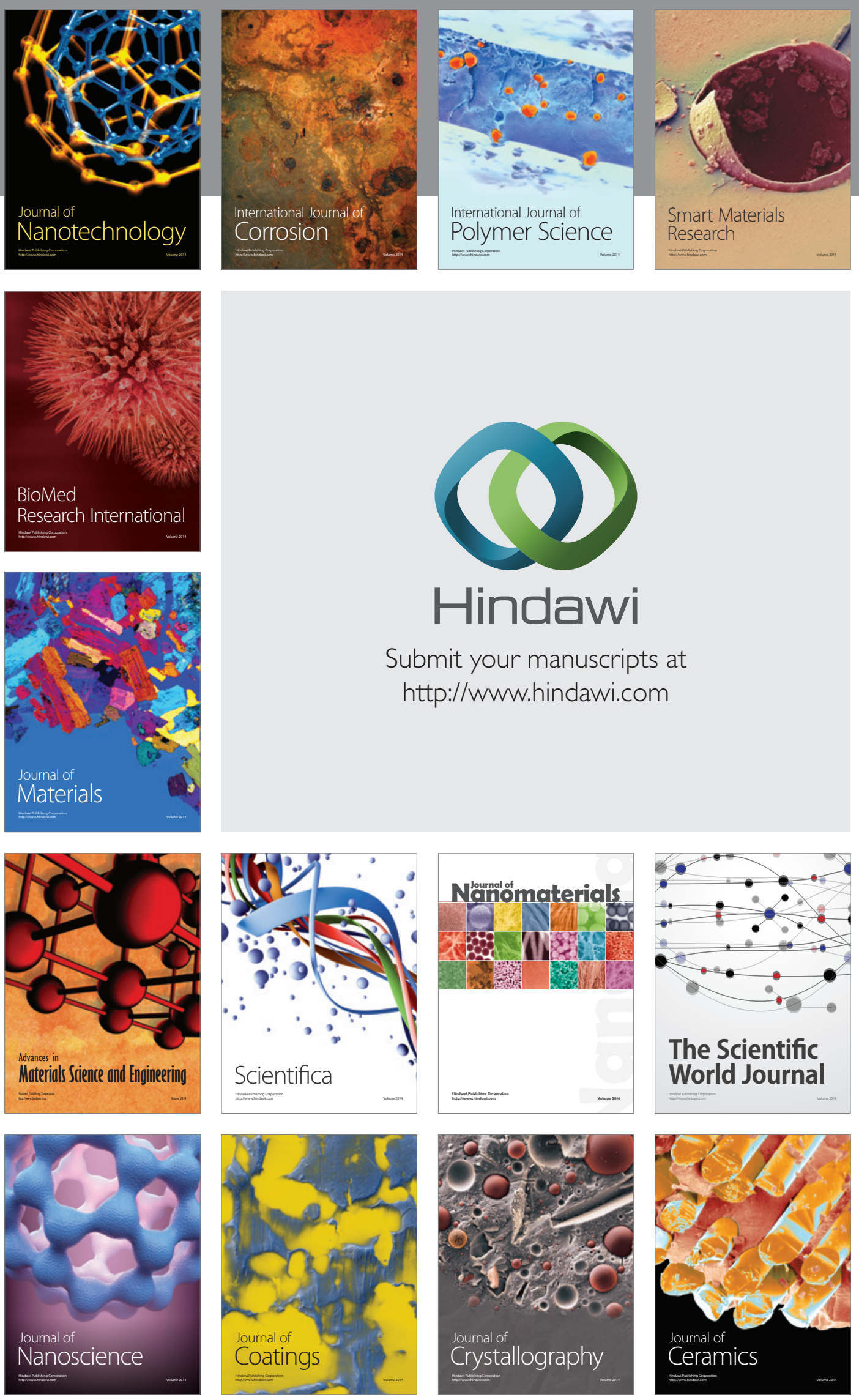

The Scientific World Journal

Submit your manuscripts at

http://www.hindawi.com

\section{World Journal}

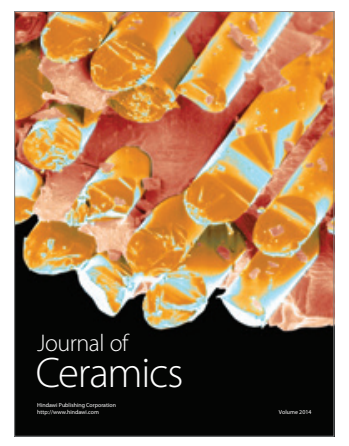

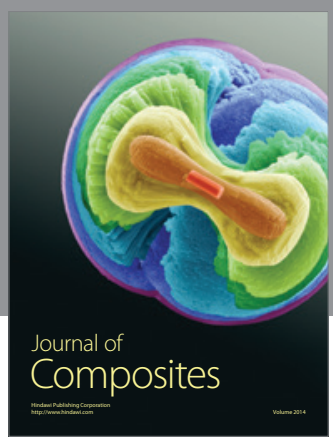
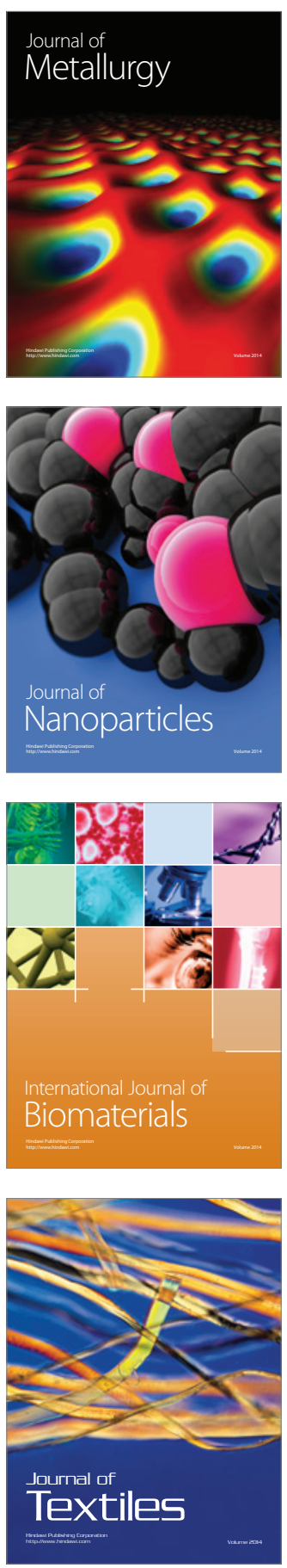